Research Article

\title{
Optimizing Regenerative Braking: A Variational Calculus Approach
}

\author{
L. Q. English (i), ${ }^{1}$ A. Mareno, ${ }^{2}$ and Xuan-Lin Chen ${ }^{3,4}$ \\ ${ }^{1}$ Department of Physics, Dickinson College, Carlisle 17013, Pennsylvania, USA \\ ${ }^{2}$ Department of Mathematics and Computer Science, Pennsylvania State University, Capital College, Middletown 17057, \\ Pennsylvania, USA \\ ${ }^{3}$ Department of Fluid Physics, Pattern Formation, and Biocomplexity, Max Planck Institute for Dynamics and Self-Organization, \\ Göttingen 37077, Germany \\ ${ }^{4}$ Department of Physics, Heilongjiang University, Harbin 150006, Heilongjiang, China
}

Correspondence should be addressed to L. Q. English; englishl@dickinson.edu

Received 1 July 2021; Accepted 9 October 2021; Published 12 November 2021

Academic Editor: Min Ye

Copyright (c) 2021 L. Q. English et al. This is an open access article distributed under the Creative Commons Attribution License, which permits unrestricted use, distribution, and reproduction in any medium, provided the original work is properly cited.

We begin by analyzing, using basic physics considerations, under what conditions it becomes energetically favorable to use aggressive regenerative braking to reach a lower speed over "coasting" where one relies solely on air drag to slow down. We then proceed to reformulate the question as an optimization problem to find the velocity profile that maximizes battery charge. Making a simplifying assumption on battery-charging efficiency, we express the recovered energy as an integral quantity, and we solve the associated Euler-Lagrange equation to find the optimal braking curves that maximize this quantity in the framework of variational calculus. Using Lagrange multipliers, we also explore the effect of adding a fixed-displacement constraint.

\section{Introduction}

Regenerative braking is a process by which some of the initial kinetic energy of a vehicle, rather than wasted as heat, is instead recovered by converting it into electrical energy which in turn is stored as chemical energy in the battery [1]. In the engineering literature, this topic is usually approached from a technological perspective [2-6]. In contrast, here we take a more simplified yet fundamental approach to the subject drawing from classical mechanics and the calculus of variations.

There has recently been some debate over the benefits of "1-pedal driving"-this is a mode where as soon as the driver of an electric vehicle (EV) releases pressure on the accelerator pedal, the electric generator is engaged, the battery charges up, and the EV begins to slow down. Tesla, for instance, makes this mode standard in all its cars. Other manufacturers, such as Volkswagen (VW), have opted to preserve the driving experience of conventional internalcombustion-engine (ICE) cars, whereby releasing the accelerator pedal allows the car to coast. Coasting means that neither the motor nor the generator are engaged, and the vehicle slows down solely due to aerodynamic drag and other friction. Some people have argued that coasting is, in fact, always best in terms of efficiency, assuming no constraints on the stopping distance [7].

In this paper [8], we start by comparing the two strategies over the same fixed displacement, and we find that aggressive regenerative braking is superior to coasting as long as the final speed is small enough. While it is true that both the electric motor and the generator do not operate at perfect efficiency and produce losses, lowering the speed faster lowers the drag force on the vehicle (relative to coasting). Thus, there exists a trade-off: regenerative braking recovers some of the initial kinetic energy (though not all of it), but the electric motor then has to use additional energy to keep the vehicle in motion. Is more energy initially stored in the battery as is later drawn from it? As we will see, the answer often is yes.

In the second part of the paper, we reformulate the question in terms of an optimization problem. Here we ask the following: what should the speed profile be that leaves the 
battery in the largest state of charge (SOC)? This recovered energy is an integral quantity, and we thus formulate the associated Euler-Lagrange equation whose solution maximizes this quantity and yields the optimal velocity as a function of time. Modeling the regenerative-braking efficiency in the simplest, most tractable way, we solve this non-linear differential equation numerically, as well as analytically in integral form. Finally, we examine the effect of adding a fixed-displacement constraint via a Lagrange multiplier.

\section{Recoverable Energy Considerations}

2.1. Coasting. In this scenario, we would like to rely solely on air drag and rolling friction to slow our EV down from an initial speed of $v_{i}$ to a final speed of $v_{f}$. Here we ignore the contributions of rolling friction-it only exhibits a weak dependence on vehicle speed [9] and should thus result in a similar energy loss when comparing coasting with regenerative braking. What is the final state of the battery relative to its initial state while coasting? It is clear that they are the same. No energy is transferred to or from the battery.

For the sake of comparison with the regenerativebraking scenario, let us calculate the accumulated displacement for this coasting process. Let us assume that the drag force is quadratic in velocity, such that

$$
F_{D}=\frac{1}{2} \rho A C_{d} v^{2}=D v^{2},
$$

where $A$ is the cross-sectional area, $\rho$ is the density of air, and $C_{d}$ is the drag coefficient, and we have combined these parameters into a single constant, $D$. This form of the drag force is valid for relatively high speeds, such as a car on a highway, but fails at low speeds. We thus arrive at the following differential equation governing coasting:

$$
m \dot{v}=-D v^{2},
$$

where $m$ is the car mass. Equation (2) has the well-known analytical solution

$$
\frac{1}{v(t)}=\frac{1}{v_{i}}+\left(\frac{D}{m}\right) t
$$

The displacement, $\Delta x=\int_{0}^{t_{c}} v(t) \mathrm{d} t$, can then be found, after a few steps.

$$
\Delta x=\frac{m}{D} \ln \left(\frac{v_{i}}{v_{f}}\right) .
$$

It is clear from equation (4) that we cannot use the quadratic-drag assumption down to a $v_{f}$ of zero.

2.2. Regenerative Braking. Instead of coasting, let us now try a different strategy: we will slow the EV down quickly from $v_{i}$ to $v_{f}$ using regenerative braking, and then we will continue to drive at $v_{f}$ until we have covered the same distance, $\Delta x$, as we did in the coasting scenario. For sake of simplicity, let us further assume that in the braking phase, the speed drops linearly in time, and that we accomplish this part in a time, $t_{r}$, as shown in Figure 1. As we will see in Section 3, this constant-acceleration strategy is not optimal and can still be improved upon, but it serves as a good starting point. The total displacement can now be expressed as

$$
\Delta x=v_{f} t_{r}+\frac{1}{2}\left(v_{i}-v_{f}\right) t_{r}+v_{f}\left(t_{f}-t_{r}\right)=\frac{1}{2}\left(v_{i}-v_{f}\right) t_{r}+v_{f} t_{f} .
$$
yields

Setting this expression equal to equation (4) for coasting

$$
t_{f}=\frac{(m / D) \ln \left(v_{i} / v_{f}\right)}{v_{f}}-\frac{v_{i}-v_{f}}{2 v_{f}} t_{r} .
$$

This $t_{f}$ must be longer than the coasting time, $t_{c}$, of course.

Next, let us look at the battery SOC during the two stages-the initial regenerative-braking stage, followed by the constant-speed stage. First, how much energy can be transferred to the battery during the first stage? Here kinetic energy is converted to electrical energy and then stored as chemical energy in the battery. Let us assume that the efficiency from kinetic to chemical-battery energy is given by $\eta$. We then have that

$$
\Delta E_{\text {batt }}^{(1)}=\eta\left(|\Delta T|-W_{\text {fric }}^{(1)}\right),
$$

with $|\Delta T|=(1 / 2) m\left(v_{i}^{2}-v_{f}^{2}\right)$. Equation (7) can also be regarded as a definition of $\eta$, the regenerative efficiency $[10,11]$. But what is $W_{\text {fric }}^{(1)}$ for this stage?

We know that $W_{\text {fric }}^{(1)}=\int F_{D} \mathrm{~d} x=D \int_{0}^{t_{r}} v^{3} \mathrm{~d} t$, where the speed is given by

$$
v(t)=v_{i}-\left(\frac{v_{i}-v_{f}}{t_{r}}\right) t,
$$

describing the constant deceleration part shown in Figure 1 (blue line). If we denote $v_{i}-v_{f}$ by $w$, expand out the cubic term, and change variables of integration, we can show that

$$
W_{\text {fric }}^{(1)}=D t_{r}\left(v_{i}^{3}-\frac{3}{2} v_{i}^{2} w+v_{i} w^{2}-\frac{1}{4} w^{3}\right) .
$$

We can then substitute equation (9) into equation (7).

Now let us turn to the second stage. Here the electric motor has to simply counteract the drag force. The work done by the drag force is given by

$$
W_{\text {fric }}^{(2)}=D v_{f}^{2} \Delta x_{2}=D v_{f}^{3}\left(t_{f}-t_{r}\right),
$$

with $t_{f}$ given by equation (6).

Assuming that the efficiency of converting chemical energy stored in the battery to electrical and then mechanical energy is $\epsilon$, we arrive at

$$
\Delta E_{\text {batt }}^{(2)}=-\frac{1}{\epsilon} W_{\text {fric }}^{(2)}
$$

where $W_{\text {fric }}^{(2)}$ is given by equation (10), and so $\Delta E_{\text {batt }}=\Delta E_{\text {batt }}^{(1)}+\Delta E_{\text {batt }}^{(2)}$. The problem is then reduced to determining the sign of $\Delta E_{\text {batt }}$. 


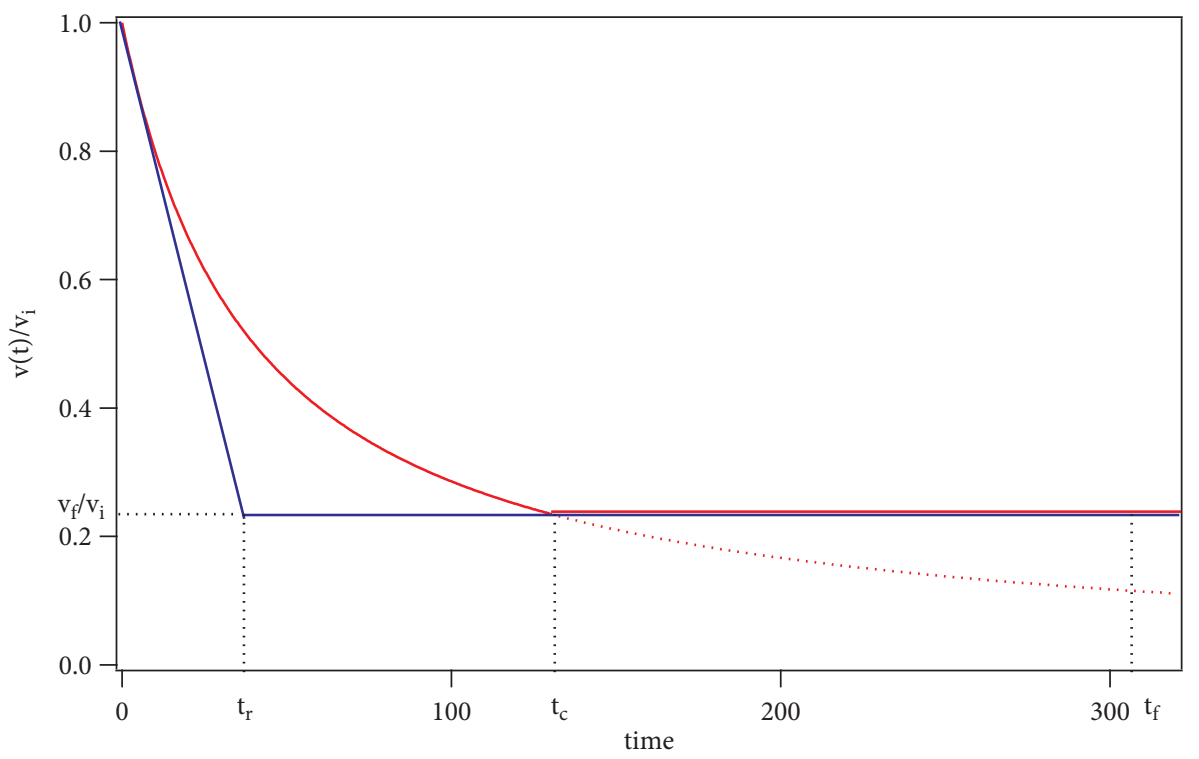

FIgURE 1: Speed as a function of time for the two processes: coasting (red line) and regenerative braking (blue line) from $v_{i}$ to $v_{f}$. The relevant times are indicated on the horizontal axis.

This expression for $\Delta E_{\text {batt }}$ is still fairly complicated and includes 7 parameters: $v_{i}, v_{f}, t_{r}, D, m, \eta$, and $\epsilon$. Let us simplify things by analyzing the limit of short $t_{r}$, such that $t_{r} \ll t_{f}$. In this limit of $t_{r} \longrightarrow 0$, the equations simplify significantly, starting with equation (6), where we can drop the second term. Furthermore, $W_{\text {fric }}^{(1)}$ in equation (9) is zero in this limit, and we arrive at

$$
\Delta E_{\text {batt }}=\frac{1}{2} m\left(v_{i}^{2}-v_{f}^{2}\right) \eta-m v_{f}^{2} \ln \left(\frac{v_{i}}{v_{f}}\right) \frac{1}{\epsilon} .
$$

In other words, we have to determine the sign of the following expression:

$$
M=\left(\frac{v_{i}^{2}}{v_{f}^{2}}-1\right) \eta-2 \ln \left(\frac{v_{i}}{v_{f}}\right) \frac{1}{\epsilon} .
$$

Equation (13) is plotted in Figure 2 as a function of speed ratio for two different sets of efficiencies. We see that for speed ratios only slightly larger than $1, M$ is negative. For larger speed ratios, $M$ always turns positive. The crossing depends sensitively on the efficiencies of the motor and generator, with lower efficiency pushing that break-even point out to larger speed ratios, $v_{i} / v_{f}$.

The conclusion is that coasting is preferable when the final target speed is not substantially below the initial speed, i.e., for instances where the desired slowdown is only moderate. For instances where a more dramatic slowdown is desired, aggressive regenerative braking wins out. A quick calculation reveals that if we want to slow down to half of our initial speed, say from $60 \mathrm{mph}$ to $30 \mathrm{mph}$, and assuming that $\eta=\epsilon$, any efficiency larger than $\eta=\epsilon=68 \%$ will favor regenerative braking.

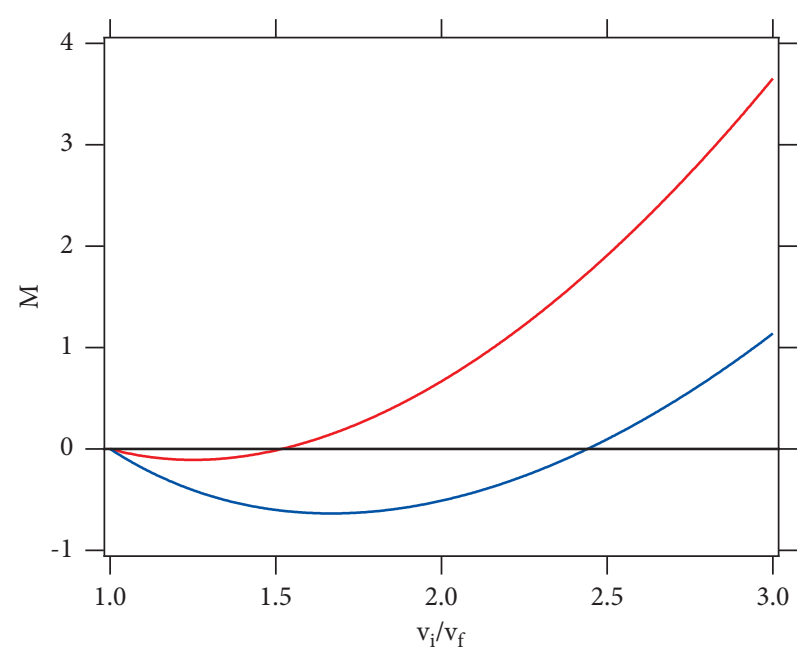

FIGURE 2: The expression $M$ of equation (13) plotted as a function of the speed ratio. The red line is for $\epsilon=\eta=0.8$, and the blue line is for $\epsilon=\eta=0.6$.

2.3. A Concrete Example. So far, the treatment has been theoretical. To make the conclusions more concrete, let us calculate actual numbers from a speed-down experiment with a Tesla (Model 3) [12]. For concreteness, consider decreasing the speed from $50 \mathrm{mph}(22.35 \mathrm{~m} / \mathrm{s})$ to $25 \mathrm{mph}$ $(11.18 \mathrm{~m} / \mathrm{s})$ and compare regenerative braking to coasting. The parameters of this vehicle are given as follows: the drag coefficient $C_{d}=0.23$, the frontal area $A=2.22 \mathrm{~m}^{2}$, and the weight of the car (with a driver) $m=1280 \mathrm{~kg}$ [13]. The density of dry air is $\rho=1.225 \mathrm{~kg} / \mathrm{m}^{3}$.

Assuming an efficiency of $\epsilon=\eta=0.75, t_{f}$ is calculated from equation (6) as 


$$
t_{f}=253.8-0.5 t_{r}
$$

Next, from equations (7) and (11) and $\Delta E_{\text {batt }}=\Delta E_{\text {batt }}^{(1)}$ $+\Delta E_{\text {batt }}^{(2)}$, we get

$$
\Delta E_{\text {batt }}=32103.7-354.7 t_{r} .
$$

According to equations (14) and (15), $t_{f}$ and $\Delta E_{\text {batt }}$ have a linear relationship with $t_{r}$, and $\Delta E_{\text {batt }}$ is positive only when $t_{r}<92 \mathrm{~s}$. Furthermore, we can also get the time of coasting $t_{c}=183 \mathrm{~s}$ from equation (3), and thus we explicitly verify the time ordering, $t_{r}<t_{c}<t_{f}$ when $\Delta E_{\text {batt }}>0$.

In an actual speed-down experiment with a Tesla Model 3 we were able to reach a minimum $t_{r}$ of around $5.0 \mathrm{~s}$. This is much below the theoretical cutoff value of $92 \mathrm{~s}$ (where $\left.\Delta E_{\text {batt }}=0\right)$, as calculated above. We have to remember, however, that calculated $t_{c}$ and $t_{r}$ are too long compared to realistic numbers, as the real dissipation is not just in the form of air drag but also includes rolling friction (tire and road) and internal friction. Nonetheless, $5 \mathrm{~s}$ is significantly below $92 \mathrm{~s}$, and it is also well below realistic coasting times. It is thus very likely that this speed-down experiment left the battery with a larger charge than what coasting would have accomplished.

\section{Optimizing Regenerative Braking}

3.1. Charging Efficiency Considerations. From the previous discussion, and in particular from equation (15), it becomes clear that without any further consideration of the charging efficiency involved, the best strategy is to brake very severely to reach the desired target speed as quickly as possible and then to remain at that constant speed for the rest of the way. This was also embodied in our examined limit of very short $t_{r}$. As we now show, a variational calculus formulation of that problem confirms this observation. In an initial attempt to shift into this framework, we seek to maximize the following functional:

$$
S=\int_{0}^{T} \mathscr{L} \mathrm{d} t
$$

where

$$
\mathscr{L}=\mathscr{L}[v, \dot{v}]=-m v \dot{v}-D v^{3} .
$$

The expression for $\mathscr{L}$ is derived from the combination of $|\mathrm{d} T|=-m v \dot{v} \mathrm{~d} t$ and $\mathrm{d} W_{\text {fric }}=D v^{3} \mathrm{~d} t$ (where $T$ denotes the kinetic energy). The associated Euler-Lagrange equation,

$$
\frac{\mathrm{d}}{\mathrm{d} t}\left(\frac{\partial \mathscr{L}}{\partial \dot{v}}\right)=\frac{\partial \mathscr{L}}{\partial v}
$$

then quickly leads to the result that $v(t)=0$.

If we think about it a bit, this result should not be unexpected. Since we have assumed that the regenerative efficiency, $\eta$, is entirely constant, it makes sense that the energetically best thing we could do is to immediately slow down to zero. If we have a constraint that we must travel a certain distance, i.e., that $\int_{0}^{T} v(t) \mathrm{d} t=d$, the analysis can be modified by adding a Lagrange multiplier, but the end result is similar: it is best to immediately slow down to a small constant speed (consistent with the distance, $d$ ).

It is clear that the culprit for this trivial answer is our assumption of constant efficiency. In reality, what EVs do when they need to slow down quickly is to blend in the physical brakes to assist the regenerative braking. The more severe the required deceleration is, the more heavily the $\mathrm{EV}$ relies on brake pads and rotors. This means that the efficiency of converting kinetic energy into battery energy goes down significantly as the braking action quickens. The battery can only accept so much power delivered to it, and this maximum power also depends sensitively on the battery SOC and temperature. Even before we reach that power limit, Ohmic losses tend to increase with charging power to reduce efficiency $[11,14]$, and friction braking is often blended in $[4,5,10]$.

How should we model these effects on efficiency? To start, it is clear that the efficiency is a monotonically decreasing function of the braking power that the battery ideally would be expected to "absorb." This power, in turn, could be approximated by the reduction in kinetic energy of the vehicle, $P \approx-\mathrm{d} T / \mathrm{d} t=-m v \dot{v}$. Thus, we can write $\eta=\eta(p)=\eta(v, \dot{v})$. We will later choose a linearly decreasing function. Such choice of $\eta(P)$, while not accounting for the full complexity, does capture the overall effect on efficiency heuristically and keeps the mathematics tractable.

3.2. Optimal Braking Curves without Distance Constraints. Informed by these considerations, we now seek to maximize the following functional:

$$
S=\int_{0}^{T} \eta(v, \dot{v})\left(-m v \dot{v}-D v^{3}\right) \mathrm{d} t
$$

The function, $\eta(v, \dot{v})$, should be close to $\eta_{0}$ for low $v \dot{v}$ and then decrease for larger $v \dot{v}$.

Let us next substitute this new L of equation (19) into the Euler-Lagrange equation, equation (18), and this returns a fairly complicated equation with several terms:

$$
\left[\frac{\partial \eta}{\partial v}-\frac{\mathrm{d}}{\mathrm{d} t}\left(\frac{\partial \eta}{\partial \dot{v}}\right)\right]\left(-m v \dot{v}-D v^{3}\right)=\eta\left(3 D v^{2}\right)-\dot{\eta} m v+\frac{\partial \eta}{\partial \dot{v}}\left(-m v \ddot{v}-m(\dot{v})^{2}-3 D v^{2} \dot{v}\right) .
$$


It is interesting to note that the first part of equation (20) is in the form of an Euler-Lagrange equation involving $\eta$ as the functional. We must now specify a functional form for $\eta$ to proceed. To keep things mathematically tractable, let us choose the following functional form for $\eta$ :

$$
\eta(v, \dot{v})=\eta_{0}+b(m v \dot{v})
$$

Here we have assumed that the efficiency drops linearly with power; the two parameters are the $y$-intercept $\eta_{0}$ and the slope, $b$. Note that $b$ here is positive, since $\dot{v}$ is negative. A side benefit of this choice for $\eta$ is that it forces the left side of equation (20) to zero, yielding

$$
\frac{\partial \eta}{\partial \dot{v}}\left(m v \ddot{v}+m(\dot{v})^{2}+3 D v^{2} \dot{v}\right)+m v \dot{\eta}-3 \eta D v^{2}=0 .
$$

From equation (21), $(\partial \eta / \partial \dot{v})=b m v$ and $(\partial \eta / \partial v)=b m \dot{v}$, and we get

$$
m v \dot{\eta}=m v\left(\frac{\partial \eta}{\partial v} \dot{v}+\frac{\partial \eta}{\partial \dot{v}} \ddot{v}\right)=b m^{2} v(\dot{v})^{2}+b m^{2} v^{2} \ddot{v} .
$$

Substituting equations (23) and (21) into equation (22) yields (after a few steps) the following differential equation:

$$
2 b m^{2} v^{2} \ddot{v}+2 b m^{2} v(\dot{v})^{2}-3 \eta_{0} D v^{2}=0 .
$$

Although not imperative, it can be helpful to nondimensionalize equation (24). For this purpose, we introduce the non-dimensional quantities $u$ and $\tau$, defined as $\tau=t / \alpha$, with $\alpha=\left(m / D v_{i}\right)$, and $u=\alpha(D / m) v=v / v_{i}$. Inserting these definitions into equation (24), we arrive at the governing equation:

$$
u^{2} u^{\prime \prime}+u\left(u^{\prime}\right)^{2}-\gamma u^{2}=0 .
$$

Here the prime indicates differentiation with respect to $\tau$, and

$$
\gamma=\frac{3 \alpha^{3} \eta_{0} D^{2}}{2 m^{3} b}=\frac{3 \eta_{0}}{2 D b v_{i}^{3}}
$$

Equation (25) represents a second-order non-linear differential equation that we can reduce to a first-order one. Dividing by $u^{2}$ and introducing $w=u^{\prime}$, we can rewrite equation (25) in the following form:

$$
\frac{\mathrm{d} w}{\mathrm{~d} u}+\frac{w}{u}=\frac{\gamma}{w}
$$

We recognize this as a Bernoulli-type equation, which can be solved to obtain

$$
w(u)=-\frac{\sqrt{2 \gamma u^{3}+k_{1}}}{\sqrt{3} u} .
$$

Here $k_{1}$ is a constant of integration that can be computed from the initial conditions as follows: since $w(1)=u^{\prime}(\tau=0)=u_{0}^{\prime}$, equation (28) leads to $k_{1}=3\left(u_{0}^{\prime}\right)^{2}-2 \gamma$.

To obtain $u(\tau)$, we remember that $u^{\prime}=w$. Thus,

$$
\int_{1}^{u} \frac{\mathrm{d} \tilde{u}}{w(\widetilde{u})}=\int_{0}^{\tau} \mathrm{d} \widetilde{\tau}
$$

and finally

$$
\tau=\int_{1}^{u} \frac{\sqrt{3} \tilde{u} \mathrm{~d} \tilde{u}}{\sqrt{2 \gamma \tilde{u}^{3}+k_{1}}}
$$

The integral on the right side of equation (30) can be evaluated in closed form (which would involve hypergeometric functions) or numerically integrated.

Alternatively, we could of course solve the original differential equation, equation (25), numerically by specifying the initial conditions $\left(u(0), u^{\prime}(0)\right)$. In either case, it is important to estimate realistic values of $\gamma$ given by equation (26). Every constant therein is straightforward with the exception of $b$. To get a reasonable estimate of this constant, we can start with maximum charging rates for the Tesla Model $3(\mathrm{SR}+)$ of about $100 \mathrm{~kW}$. It is likely that for this vehicle, the maximum regenerative power is software-limited to the somewhat lower threshold of around $75 \mathrm{~kW}$. In our mathematical model, of course, the efficiency goes down linearly with power. Let us assume that we reduce $\eta$ to $\eta_{0} / 2$ for $75 \mathrm{~kW}$. This then implies a value of $b=5 * 10^{-6} \mathrm{~s} / \mathrm{J}$, which yields $\gamma=67.5$ for $\eta_{0}=0.75$ and $v_{i}=50 \mathrm{mph}$.

Figure 3(a) shows some typical numerical solutions obtained with Mathematica using the "NDSolve" command [15]. For the blue trace, we set $\gamma$ to 40 , and for the red trace, $\gamma=70$. The initial conditions were $u(0)=1$ for both, and $u^{\prime}(0)$ was adjusted to obtain the same stopping time, $\tau_{f}=0.14$ (defined by $\left.v\left(\tau_{f}\right)=0\right)$. For the red trace, this implies $u^{\prime}(0)=-7.01$, and for the blue, $u^{\prime}(0)=-5.65$. It should be noted that we get indistinguishable curves by using equation (30) and inverting the resulting $\tau(u)$.

Also shown for comparison in Figure 3(a) is the speed profile for a run where the extracted power is constant in time (black trace), with that constant adjusted to again yield the same stopping time. A straightforward calculation reveals that for constant power, $v(t)=\sqrt{v_{0}^{2}-\kappa t}$.

When comparing the red and black traces, it is evident that the optimal solution is one where initially, at high speeds, more power is extracted than for lower speeds. This makes sense, since the goal is to minimize losses from air drag, and drawing out more energy from the available kinetic energy during the initial phase is advantageous.

Notice also that, according to equation (26), when the parameter $\gamma$ is raised, $b$ decreases, assuming the same EV is driven (identical $m, D, \eta_{0}$ ) and the same starting speed, $v_{i}$, is used. For smaller slope, $b$, governing $\eta(P)$, the regenerative braking can be made more severe without incurring additional efficiency penalties. This explains the more negative overall acceleration seen in the red trace at the start, when compared with the blue trace.

Finally, Figure 3(b) plots the same data as an acceleration graph, $u^{\prime}$ versus $\tau$. As before, the $\gamma=70$ solution appears in red, $\gamma=40$ in blue, and the constant-power curve in black. For all curves, $u^{\prime}$ diverges to negative infinity as $u \longrightarrow 0$. However, it is also apparent that the optimal solutions for both values of $\gamma$ 


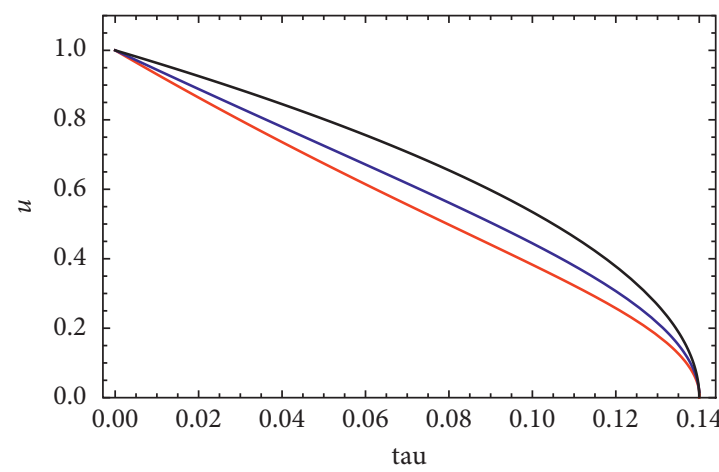

(a)

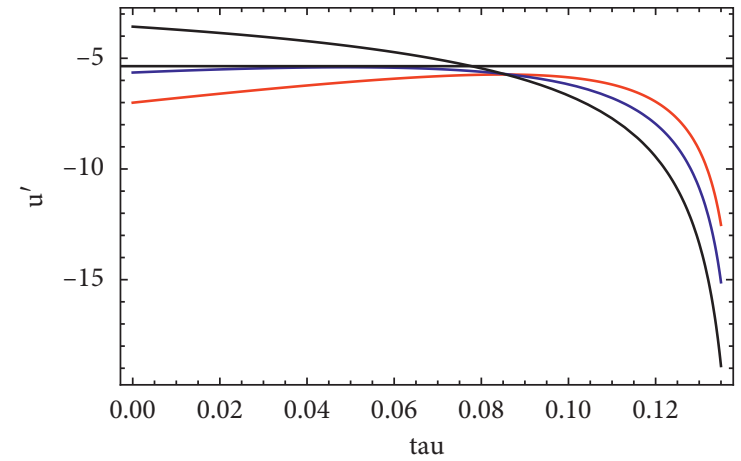

(b)

Figure 3: Numerical solutions for $u(\tau)$ such that the stopping time is 0.14 . (a) Blue curve shows $\gamma=40$, and red curve shows $\gamma=70$. For comparison, the black trace depicts the constant-power curve. (b) The same data plotted as an acceleration graph. All accelerations become extremely negative near $\tau=0.14$, so we plot only to 0.13 to highlight to essential differences between the traces.

feature more negative accelerations at the beginning and a less negative acceleration towards the end, compared to the constant-power curve. We again see that air-drag considerations nudge the optimal solutions in the direction of reducing speed at the beginning as much as possible.

This feature of the optimal solution is also present when the vehicle does not come to a stop but reaches a non-zero final speed. Mathematically, we need only modify the endpoint condition to $v(T)=v_{f}$. Figure $4(\mathrm{a})$ depicts the three solutions of the previous figure but now for a final (reduced) speed of $u\left(\tau_{f}\right)=0.5$, with $\tau_{f}=0.1$. In Figure $4(\mathrm{~b})$, the same data are plotted as an acceleration graph that accentuates the differences and again showcases the strategy of making the acceleration as negative as possible at small times (red trace).

These optimized acceleration curves in Figure 4(b) can be compared to accelerometer data from a real test drive in a Tesla Model 3 where the speed was reduced from $50 \mathrm{mph}$ to $25 \mathrm{mph}$. This measurement is shown in Figure 5. We see that both the magnitude of acceleration, $\left|u^{\prime}\right|$, and the recovered power are not constant but largest at short times. In that respect, the result resembles the red curve of Figure 4(b).

We conclude this section by circling back to the original question: how much energy is added or drawn from the battery after performing braking actions according to these optimized solutions? $\Delta E_{\text {batt }}$ is, in fact, given by the functional of equation (19). If this integral, when evaluated for the numerical solutions found earlier, is positive, the battery gains charge during the examined time interval. Casting this definite integral in terms of the non-dimensional variables, $u$ and $\tau$, we get that $\eta\left(u, u^{\prime}\right)=\eta_{0}\left(1+(3 / 2 \gamma) u u^{\prime}\right)$, and

$$
\Delta E_{\text {batt }}=\eta_{0}\left(m v_{i}^{2}\right) \int_{0}^{\tau_{f}}\left(1+\frac{3}{2 \gamma} u u^{\prime}\right)\left(-u u^{\prime}-u^{3}\right) \mathrm{d} \tau .
$$

We use the "NIntegrate" command in Mathematica to evaluate this integral for the traces in Figure 3 (where $\left.\tau_{f}=0.14\right)$. We thus obtain $0.315 m v_{i}^{2}$ for the red trace and $0.293 m v_{i}^{2}$ for the blue trace. Both integrals are therefore positive and less than $\eta_{0}\left(T_{i}-T_{f}\right)$. Both solutions are also improvements over the constant-power curve; when compared to the red trace, it recovers roughly $2 \%$ less energy.

Similarly, we can compare the optimal braking curves in Figure 4 to the constant acceleration case considered earlier in Section 2, where the speed drops linearly with time. When we do this for the $\gamma=70$ curve (red trace), for instance, we find that the optimal solution recovers $0.226 m v_{i}^{2}$, which is only about $0.1 \%$ more energy than constant-acceleration braking. In a sense, this observation retroactively validates the initial, more simplified approach.

3.3. Adding a Distance Constraint with a Lagrange Multiplier. We can ask a slightly modified question: what is the optimal braking curve, $v(t)$, that connects the two points, $v(0)=v_{i}$ and $v(T)=v_{f}$, given that the car must cover a specified distance? Notice that we now have added another condition at the end. We would like to consider curves of equal displacement, $d=\int_{0}^{T} v \mathrm{~d} t$. Variational calculus tells us that we now need to make the following integral stationary:

$$
\int_{0}^{T}(\mathscr{L}+\lambda v) \mathrm{d} t
$$

where $\lambda$ is called the Lagrange multiplier and is related to the displacement. The Euler-Lagrange equation now reads

$$
\frac{\mathrm{d}}{\mathrm{d} t}\left(\frac{\partial \mathscr{L}}{\partial \dot{v}}\right)=\left(\frac{\partial \mathscr{L}}{\partial v}\right)+\lambda
$$

Following similar steps as before, we thus arrive at the modified non-dimensional governing equation:

$$
u^{2} u^{\prime \prime}+u\left(u^{\prime}\right)^{2}-\gamma u^{2}-\kappa \lambda=0
$$

with $\kappa=\left(\gamma / 3 \eta_{0} D v_{i}^{2}\right)$ such that $\kappa \lambda$ is non-dimensional.

Employing the same analytical technique of reducing equation (34) to a first-order equation yields

$$
\tau=\int_{1}^{u} \frac{\sqrt{3} \widetilde{u} \mathrm{~d} \widetilde{u}}{\sqrt{2 \gamma \tilde{u}^{3}+6 \kappa \lambda+k}}
$$

with $k=3\left(u_{0}^{\prime}\right)^{2}-2 \gamma-6 \kappa \lambda$. 


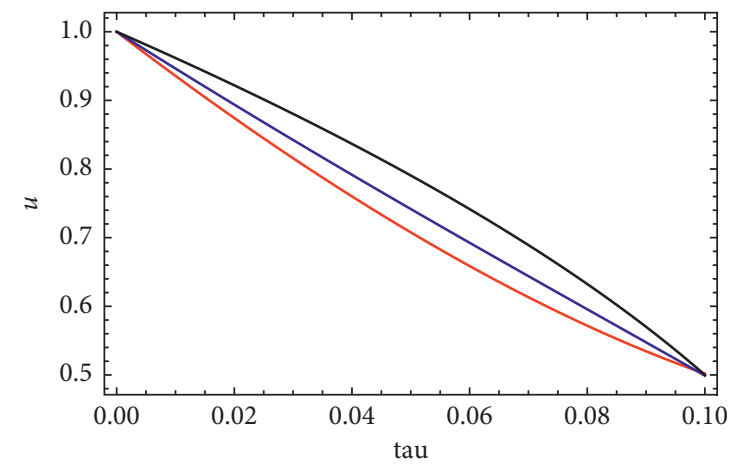

(a)

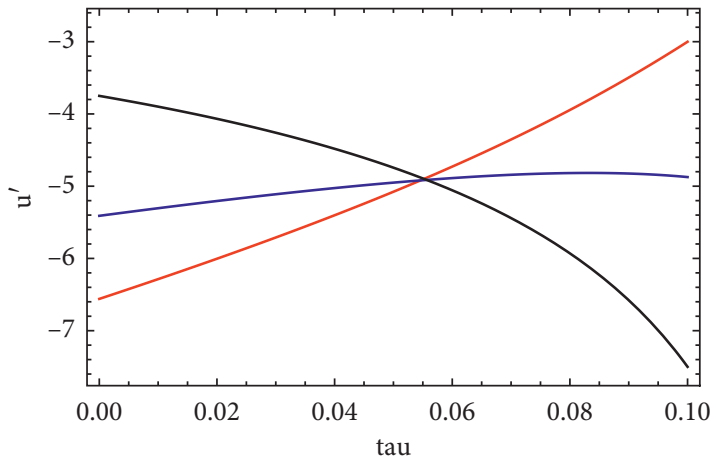

(b)

Figure 4: Numerical solutions for $u(\tau)$ such that $u(0.1)=0.5$. (a) Blue curve shows $\gamma=40$, and red curve shows $\gamma=70$. For comparison, the black trace depicts the constant-power curve. (b) The same data plotted as acceleration versus time. The differences between the various solutions are visually accentuated.

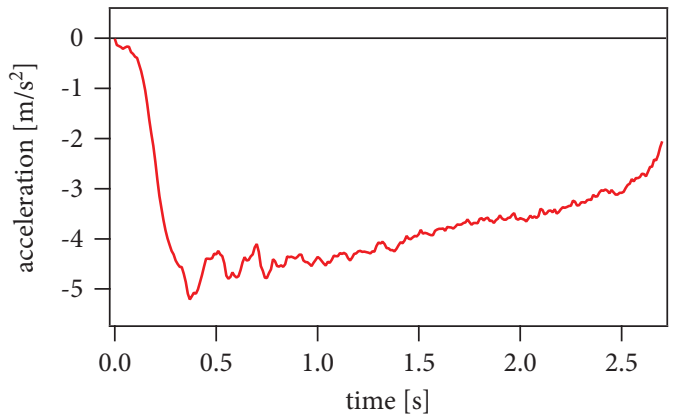

Figure 5: Accelerometer data from a test drive in a Tesla Model 3 (SR+) where the speed was reduced from $50 \mathrm{mph}$ to $25 \mathrm{mph}$ using full regenerative braking. We see that the acceleration is indeed largest initially when the speed is close to $50 \mathrm{mph}$.

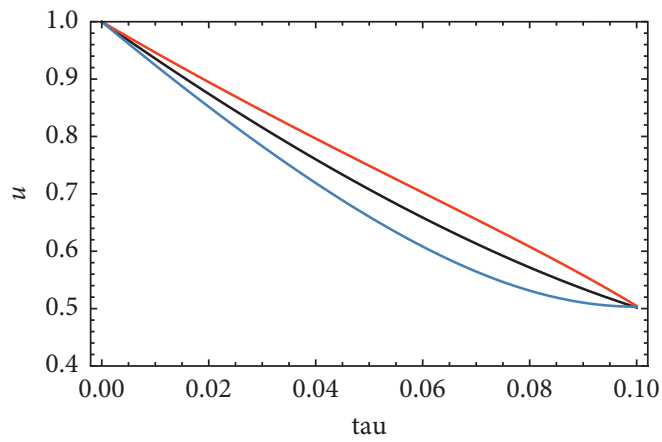

Figure 6: The optimal braking curves for three different values of the Lagrange multiplier $\kappa \lambda=\lambda^{*}$. The black, red, and blue curves show the results for $\lambda^{*}=0,19.2$, and -19.2 , respectively, and $\gamma=70$.

Figure 6 shows the effect of the distance constraint. The three traces correspond to three different values of the Lagrange multiplier and thus braking distances, with $\gamma$ set to 70 . We know that $d=\int v \mathrm{~d} t=(m / D) \int u \mathrm{~d} \tau$. Therefore, to find the actual distance traveled, we numerically integrate the traces in Figure 6. Then, using the values in Section 2.3, we obtain $d=307,295$, and $282 \mathrm{~m}$, respectively, for the red, black, and blue traces. Furthermore, $T=\alpha \tau_{f}=\alpha(0.1)=18.3 \mathrm{~s}$. The recovered energies for the red, black, and blue traces, respectively, are $0.2243 m v_{i}^{2}, 0.2260 m v_{i}^{2}$, and $0.2245 m v_{i}^{2}$. Not surprisingly, the optimal solution without displacement constraint (black trace) outperforms the other two solutions with the Lagrange multipliers.

\section{Conclusion}

We started by comparing two braking strategies-coasting and regenerative braking - and found that it is advantageous 
to employ aggressive regenerative braking for cases where the ratio of initial to final speeds is above a derived threshold that depends sensitively on the efficiencies of the motor and generator. In this analysis, we chose a simplified assumption of a constant regenerative braking acceleration. We then refined the analysis using variational calculus to optimize the functional form of the braking curves. More specifically, we derived acceleration profiles, $a(t)$, that maximize battery SOC, accounting for reduced battery-charging efficiencies with braking power. The optimal braking strategy was derived by finding both numerical and analytical (integral form) solutions to the associated Euler-Lagrange equation. Finally, we explored the effect of introducing the constraint of fixed displacement (distance traveled) via a Lagrange multiplier.

Not unexpectedly, one lesson from the optimal braking curves obtained in this manner is that it pays to make the acceleration as negative as possible at the beginning, in an effort to reduce air-drag losses as much as possible (without cutting into charging efficiencies too severely). The derived solutions thus balance this inherent trade-off in a way that recovers the most energy. To keep the problem mathematically tractable, here we used a linear charging efficiency model, but this model could be refined in a straightforward way in future work, since the Euler-Lagrange equations were derived in fair generality and could be explicitly written for any efficiency model.

\section{Data Availability}

Data were derived and taken by authors and are displayed in graphical form. The numerical data used to support the findings of this study are available from the corresponding author upon request.

\section{Disclosure}

An earlier preprint of this paper was submitted to arXiv.org, retrievable at https://arxiv.org/pdf/2106.14686.pdf.

\section{Conflicts of Interest}

The authors declare that they have no conflicts of interest.

\section{Acknowledgments}

For financial help with publication costs, LQE acknowledges support from Dickinson College's Research \& Development Committee, AM acknowledges support from Penn State Harrisburg, and XC acknowledges support from Heilongjiang University.

\section{References}

[1] S. J. Clegg, A Review of Regenerative Braking Systems, Institute of Transport Studies, University of Leeds, Leeds, UK, 1996.

[2] G. Xu, W. Li, K. Xu, and Z. Song, "An intelligent regenerative braking strategy for electric vehicles," Energies, vol. 4, no. 9, pp. 1461-1477, 2011.
[3] C. Qiu and G. Wang, "New evaluation methodology of regenerative braking contribution to energy efficiency improvement of electric vehicles," Energy Conversion and Management, vol. 119, pp. 389-398, 2016.

[4] B. Xiao, H. Lu, H. Wang, J. Ruan, and N. Zhang, "Enhanced regenerative braking strategies for electric vehicles: dynamic performance and potential analysis," Energies, vol. 10, no. 11, p. $1875,2017$.

[5] W. Liu, H. Qi, X. Liu, and Y. Wang, "Evaluation of regenerative braking based on single-pedal control for electric vehicles," Frontiers of Mechanical Engineering, vol. 15, no. 1, pp. 166-179, 2020.

[6] H. Abbas, Y. Kim, J. Siegel, and D. Rizzo, "Synthesis of pontryagin's maximum principle analysis for speed profile optimization of all-electric vehicles," Journal of Dynamic Systems, Measurement, and Control, vol. 141, p. 071004, 2019.

[7] J. Sensiba, "Regenerative braking vs. coasting: volkswagen id.4 gets it right," 2020, https://cleantechnica.com/2020/12/15/ regenerative-braking-vs-coasting-volkswagen-id-4-gets-it-right/.

[8] L. Q. English, A. Mareno, and X.-L. Chen, "Optimizing regenerative braking-a variational calculus approach," 2021, http://arxiv.org/abs/2106.14686.

[9] J. Katz, Automotive Aerodynamics, Wiley, Hoboken, NJ, USA, 2016.

[10] V. Verma, "A review of regenerative braking in hybrid vehicles," JETIR, vol. 2, p. 65, 2015.

[11] M. T. Von Srbik and R. F. Martinez-Botas, "Vehicle optimisation for regenerative brake energy maximisation," in Sustainable Vehicle Technologies, Woodhead Publishing, Sawston, UK, 2012.

[12] https://www.tesla.com/model3.

[13] A. Mohan, S. Sripad, P. Vaishnav, and V. Viswanathan, "Trade-offs between automation and light vehicle electrification," Nature Energy, vol. 5, no. 7, pp. 543-549, 2020.

[14] A. Genovese, F. Ortenzi, and C. Villante, "On the energy efficiency of quick DC vehicle battery charging," WEVJ, vol. 7, Article ID 0570, 2015.

[15] Wolfram Research Inc, Mathematica, Version 12, Wolfram Research, Inc., Champaign, IL, USA, 2021. 